\title{
Evaluation of Dose Calculation Algorithms of Isogray Treatment Planning System Using Measurement in Heterogeneous Phantom
}

\author{
Asiyeh Golestani, ${ }^{1}$ Mohammad Houshyari, ${ }^{2}$ Ahmad Mostaar, ${ }^{1, *}$ and Ali Jabbari Arfaie ${ }^{3}$ \\ ${ }^{1}$ Department of Medical Physics and Biomedical Engineering, School of Medicine, Shahid Beheshti University of Medical Sciences, Tehran, IR Iran \\ ${ }^{2}$ Department of Radiotherapy, Shohada-e-Tajrish Hospital, School of Medicine, Shahid Beheshti University of Medical Sciences, Tehran, IR Iran \\ ${ }^{3}$ Shohada-e-Tajrish Hospital, Shahid Beheshti University of Medical Sciences, Tehran, IR Iran \\ "Corresponding author: Ahmad Mostaar, Department of Medical Physics and Biomedical Engineering, School of Medicine, Shahid Beheshti University of Medical Sciences, \\ Tehran, IR Iran. Tel/Fax: +98-2122439941, E-mail: a_mostaar@yahoo.com
}

Received 2015 July 11; Accepted 2015 September 12.

\begin{abstract}
Background: Radiotherapy is a high-energy ionizing radiation treatment for some kinds of cancers. Accuracy and the quality of radiotherapy treatment planning systems depend on the type of dose calculation algorithms that are utilized. There are uncertainties in the calculation of dose distribution, especially in a heterogeneous situation.

Objectives: This study calculated and compared dose with model-based algorithms (superposition, collapsed cone convolution (CCC) and fast Fourier transform (FFT) and a measure-based algorithm (Clarkson in two modes: heterogeneity active and inactive). Materials and Methods: A heterogeneous phantom was used based on a semi-anthropomorphic phantom CIRS thorax 002 LFC. All of the tests were planned according to IAEA TEC-DOC 1583. All measurements were performed with a $6 \mathrm{MV}$ photon beam of a linear accelerator (ELEKTA Compact) and Farmer ionization chamber. Five methods were utilized to calculate dose and were compared with measurement results as the gold standard.

Results: In the homogeneity media all algorithms had good accuracy and dose difference was below 3\%, but in the inhomogeneity situation dose difference increased and in some cases did not achieve agreement criteria. The Superposition algorithm overall has minimum deviations in all cases. However in some cases CCC had better accuracy. The Clarkson algorithm had maximum differences, especially when inhomogeneity correction was inactive.

Conclusions: Dose calculation algorithms applied in radiotherapy treatment planning systems have different accuracy. Modelbased algorithms have a better accuracy over measurement-based algorithms such as Clarkson. In the presence of large inhomogeneity, it is strongly recommended to activate manual inhomogeneity correction.
\end{abstract}

Keywords: Quality Assurance, Dose Calculation Algorithms, Treatment Planning Systems

\section{Background}

Radiotherapy is treatment with high-energy ionizing radiation for some kinds of cancers. In this procedure the prescribed dose of radiation was applied to damage cancer cells and stop them from growing and dividing. A high degree of accuracy regarding the delivery dose is one major requirement of radiotherapy. According to the international commission on radiation units and measurements, ICRP, the overall accuracy of the absorbed dose required to control a tumour and to avoid complications is 5\% (1). Other groups proposed $3 \%-4 \%$ for uncertainty in delivery dose (2-6). To achieve this level of accuracy, it is necessary to audit all steps of the dose calculation: beam data, dose calculation algorithm and so on. The IAEA has developed a practical test based on TRS430 to verify the accuracy of a dissymmetry system. In radiotherapy, monitor units (MU) or beam-on time are provided to deliver actual dose to a tumour. Error in calculation MU causes dose error and a curve in the treatment. Nowadays, a three-dimensional treatment planning system (TPS) has become common in radiotherapy and therefore verification methods for dose calculation have changed (7). Dose calculation algorithms can be broadly classified. One of the major differences between various dose calculation algorithms is the approximation of the mass stopping power $(8,9)$. Although Monte Carlo is the most accurate algorithm for dose calculation, it requires a long processing time (10-12). Therefore, other algorithms such as Clarkson and point kernel are used. There are uncertainties in the calculation of dose distribution especially in a heterogeneous situation, small field, or an interface region because of electronic disequilibrium. Over the last decade, pencil beam (PB) algorithms have become the standard for dose calculation algorithms in radiotherapy. Although it has acceptable accuracy and calculation speed, certain limitations of PB algorithms and higher accuracy can be addressed through some kernel-based mod- 
els (8).

A convolution-superposition algorithm improved estimation of dose in an electronic equilibrium region. A pencil beam convolution (PBC) algorithm considers a narrow pencil beam and uses field intensity fluencies with the kernel that describes dose deposition around the primary photon pencil beam in water.

This study focuses on the dosimetric audit of the Isogray TPS (Dosisoft company) and investigation of the accuracy of its dose calculation algorithms.

\section{Objectives}

For this purpose an anthropomorphic phantom was used with a set of clinical test cases prepared by the IAEA that covers some typical clinical radiation techniques in three-dimensional radiotherapy.

\section{Materials and Methods}

All measurements were performed in a hospital with an accelerator (ELEKTA Compact) and photon having a low energy ( $6 \mathrm{MV}$ ) beam. Measurements were performed by an ionization chamber.

\subsection{Phantom}

This study used a heterogeneous phantom based on a semi-anthropomorphic phantom CIRS thorax 002 LFC. This phantom is used for clinical test measurement of the chest wall and breast. The phantom was designed from tissue, bone and lung equivalent materials and it has holes for dose measurement by an ionization chamber. It consists of a $15 \mathrm{~cm}$ thick slice and 15 standard slices with $1 \mathrm{~cm}$ thickness. The $30 \mathrm{~cm} \times 20 \mathrm{~cm}$ diameter body of Plexiglas (Perspex) has a mass density of $1.17 \mathrm{~g} / \mathrm{cm}^{3}$ and relative electron density of 1.003. There is also a cylinder of Teflon as bony vertebra and special fiber as lung that is applied with a relative electron density 1.506 and 0.207 , respectively. The phantom contains 10 holes to hold interchangeable rod inserts for an ionization chamber. The holes are numbered as shown in Figures 1 and 2. However the phantom has a limited number of available positions for dose measurements; it has slabs in between that accommodate a film dosimeter and measure dose in two dimensions. The phantom was scanned using a computed tomography (CT) scanner (Somatom Emotion 16-Slice, Siemens) that was calibrated using a special calibration agency. The CT images of the phantom in a DICOM format were transferred to the TPS. These images were used for planning the clinical tests.

Electron density relative to the $\mathrm{CT}$ number and maximum difference between $\mathrm{CT}$ numbers for same relative electron density was 20 Hounsfield units (Hus) (Figures 3 -10).

\subsection{Clinical Test Cases}

A set of clinical test cases as advised by IAEA TEC-DOC 1583 (13) were used to verify the range of basic treatment techniques applied in clinical practice. A detailed description of test cases and measurement points are given in Table 1.

Eight cases and thirty points comprised the total number of measurements. The plan was for test cases to deliver a 2 Gy dose to the reference point in each case and then the calculated dose was obtained by different algorithms at other points. All of the measurements were performed by an ionization chamber. Due to lack of MLC, test case no. 5 was not performed.

\subsection{Dose Calculation Algorithms}

Dose calculations were performed for some standard clinical tests with different algorithms to evaluate accuracy; the results were compared to measurements as a gold standard. The evaluated algorithms in this study were divided into two categories:

A) Measurement-based algorithm that includes Clarkson (two types: if manual heterogeneity correction is active named Clarkson* and if as default it is inactive and named Clarkson).

B) Model-based algorithms using point kernel convolution that includes fast-fourier transform (FFT), superposition and collapsed cone convolution (CCC).

These models account for density verification approximately for electrons and photons in lateral.

\subsection{Measurements}

All measurements were carried out on an Elekta accelerator (Compact model, Elekta Oncology Systems) operating with a photon energy of $6 \mathrm{MV}$. The $6 \mathrm{MV}$ photon beam was investigated in this study since higher energy photon beams have a less inhomogeneity correction effect (14).

For dose measurements in the phantom, a Farmer type ionization chamber (A19, Standard Imaging Co, USA) with electrometer (Supermax, Standard Imaging Co, USA) were employed. The chamber and electrometer were calibrated at secondary standard dosimetry laboratory (SSDL). For all measurement points, the absorbed dose was determined from ionization chamber measurements using the IAEA TRS 398 dosimetry code of practice (15). Pressure and temperature in each measurement condition were also measured. 


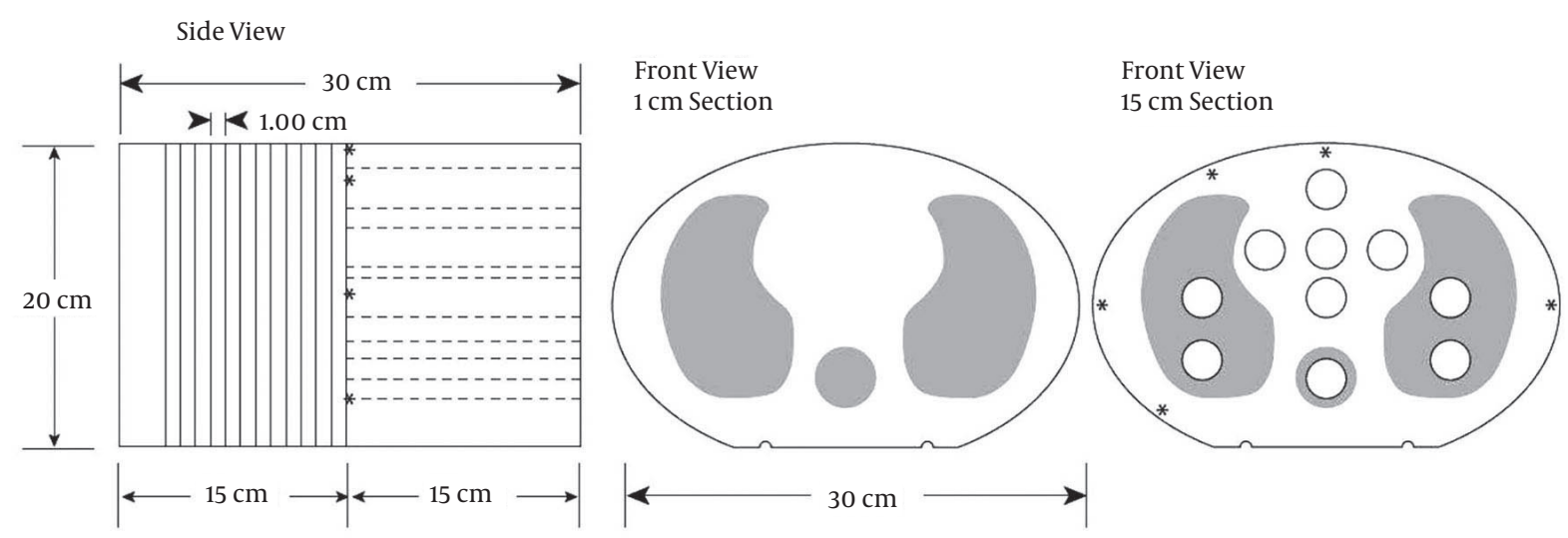

Right, Ionization chamber measurements are performed in the $15 \mathrm{~cm}$ slice; left, standard slice (13).

Figure 2. Position of Measurement Points in Phantom

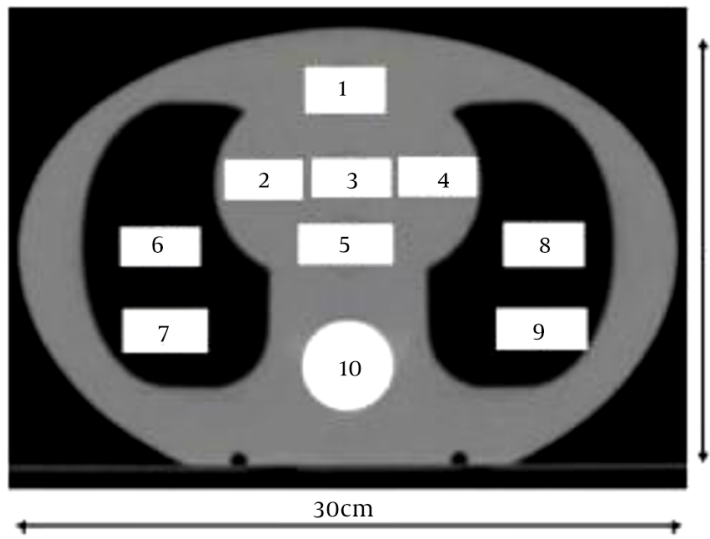

Plugs number 1,2,3, 4 and 5 are tissue equivalent material, 6, 7,8 and 9 are equivalent lung material and 10 is bone equivalent material (13).

\subsection{Analysis of the Results}

IAEA TRS (430) determines criteria for dose measured $\left(D_{\text {meas }}\right)$ and dose TPS calculated $\left(D_{\text {cal }}\right)$ such that differences are normalized to the dose measured at the reference point $\left(D_{\text {meas, ref }}\right)$ for each test case (16) (Equation 1$)$.

$\operatorname{Error}(\%)=\left(\frac{D_{\text {cal }}-D_{\text {meas }}}{D_{\text {meas }, \text { ref }}}\right) \times 100$

\section{Results}

In the homogenous media, all of the verification doses determined by algorithms (Clarkson, Clarkson*, Superposition, CCC, FFT) approximately have a highly acceptable

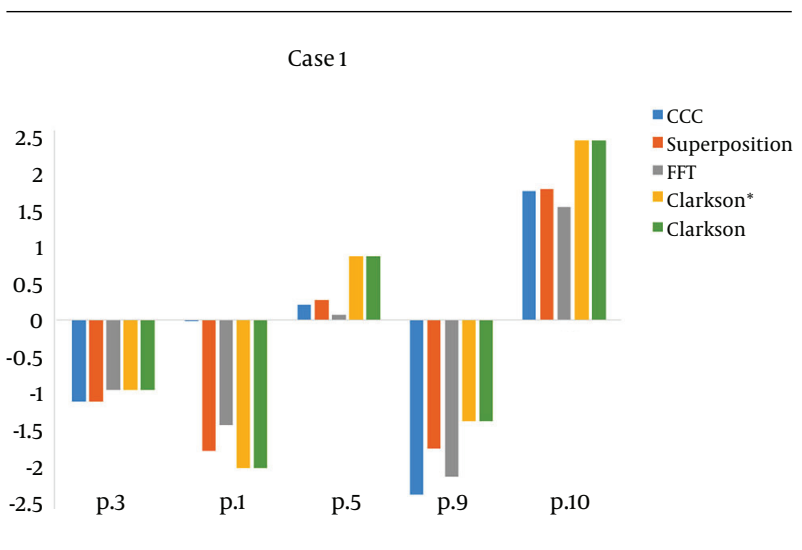

Figure 3. Difference Between Calculation and Measurement by Different Algorithms in Test Case No. 1

degree of agreement between calculations and measurements. For case 1, a single square field, all of the algorithms had acceptable accuracy and their errors were less than the agreement criteria ( $\pm 2 \%$ ). For point 9 in lung equivalent material, the calculated dose was overestimated, however, it was less than the agreement criteria. For point 10 in bone equivalent material, the calculated dose was underestimated. For all algorithms the amount of MU/D was equal to 1.15 .

The Clarkson* and Clarkson algorithms had more errors (than) the dose in the tangential beam (case 2). All of algorithms were in compliance with agreement criteria $( \pm$ $3 \%$ ). The accuracy of the model-based algorithms (CCC, Superposition and FFT) were equal, and Clarkson* and Clarkson were also equal. 
Table 1. Agreement Criteria are Listed for Each Test Case

\begin{tabular}{|c|c|c|c|c|}
\hline No. of Case & Description of Test & Reference Point & Measure Point(s) & Agreement Criteria, \% \\
\hline \multirow[t]{6}{*}{1} & Single field, $S S D=100$, angle Collimator and Gantry $=0^{\circ}$, size of field $=10 \times 10$ & 3 & & \\
\hline & & & 3 & 2 \\
\hline & & & 9 & 4 \\
\hline & & & 10 & 3 \\
\hline & & & 1 & 2 \\
\hline & & & 5 & 2 \\
\hline \multirow[t]{2}{*}{2} & Oblique incidence, lack of scattering and tangential field, $S S D=100$, angle Gantry $=90^{\circ}$, angle Collimator: depended to wedge & 1 & & \\
\hline & & & 1 & 3 \\
\hline \multirow[t]{2}{*}{3} & Significant blocking of the field corner. $S S D=100$, technique: $\mathrm{SAD}$. angle Collimator $=45^{\circ}$, filed size $14 \times 14$ down to $8 \times 10$ & 3 & & \\
\hline & & & 3 & 3 \\
\hline \multirow[t]{16}{*}{4} & Four field box, technique: SAD. Set-up at point: 5 & 5 & & \\
\hline & & & $5\left(0^{\circ}\right)$ & 2 \\
\hline & & & $5\left(90^{\circ}\right)$ & 3 \\
\hline & & & $5\left(180^{\circ}\right)$ & 3 \\
\hline & & & $5\left(270^{\circ}\right)$ & 3 \\
\hline & & & $\sum$ & \\
\hline & & & $6\left(0^{\circ}\right)$ & 4 \\
\hline & & & $6\left(90^{\circ}\right)$ & 3 \\
\hline & & & $6\left(180^{\circ}\right)$ & 4 \\
\hline & & & $6\left(270^{\circ}\right)$ & 3 \\
\hline & & & $\sum$ & \\
\hline & & & $10\left(0^{\circ}\right)$ & 3 \\
\hline & & & $10\left(90^{\circ}\right)$ & 4 \\
\hline & & & $10\left(180^{\circ}\right)$ & 3 \\
\hline & & & $10\left(270^{\circ}\right)$ & 4 \\
\hline & & & $\sum$ & \\
\hline \multirow[t]{3}{*}{5} & MLC block of clinder: $10 \mathrm{~cm}$, technique: SAD, set-up at point: 2 , angle Gantry: $0^{\circ}$, angle Collimator: $0^{\circ}$ & 2 & & \\
\hline & & & 2 & 3 \\
\hline & & & 7 & 4 \\
\hline \multirow[t]{4}{*}{6} & $\begin{array}{l}\text { L-shaped field with oblique incidence. Set-up at point:5, technique: SAD, angle Collimator: } 0^{\circ} \text {, angle Gantry: } 45^{\circ} \text {, filed size } 10 \times 20 \text { down to } 6 \\
\qquad \times 12\end{array}$ & 3 & & \\
\hline & & & 3 & 3 \\
\hline & & & 7 & 5 \\
\hline & & & 10 & 5 \\
\hline \multirow[t]{5}{*}{7} & Plan with asymmetric fields and wedge, technique: SAD. set-up at point: 3 & 5 & & \\
\hline & & & $5\left(0^{\circ}\right)$ & 2 \\
\hline & & & $5\left(90^{\circ}\right)$ & 4 \\
\hline & & & $5\left(270^{\circ}\right)$ & 4 \\
\hline & & & $\sum$ & \\
\hline \multirow[t]{5}{*}{8} & Plan with non-coplanar field. technique: SAD, set-up at point: 5 & 5 & & \\
\hline & & & $5\left(30^{\circ}\right)$ & 3 \\
\hline & & & $5\left(90^{\circ}\right)$ & 3 \\
\hline & & & $5\left(270^{\circ}\right)$ & 3 \\
\hline & & & $\sum$ & \\
\hline
\end{tabular}

${ }^{\mathrm{a}}$ The case No. 5 was not performed because of lack of MLC in our radiotherapy center.

\subsection{In Case 3}

In this test, all the algorithms overestimated the dose in the field with a wedge, although all of the results in the range were acceptable. The amount of $(M U / D)=1.185$ for measurement-base algorithms and for model- based algo- rithms was equal to 1.181 .

In the four fields box test (case 4), the reference point was in the Plexiglas, point 10 in the bone equivalent and the central axis in the vertical beams. Point 6 was in the lunge equivalent material and the central axis in the lateral 


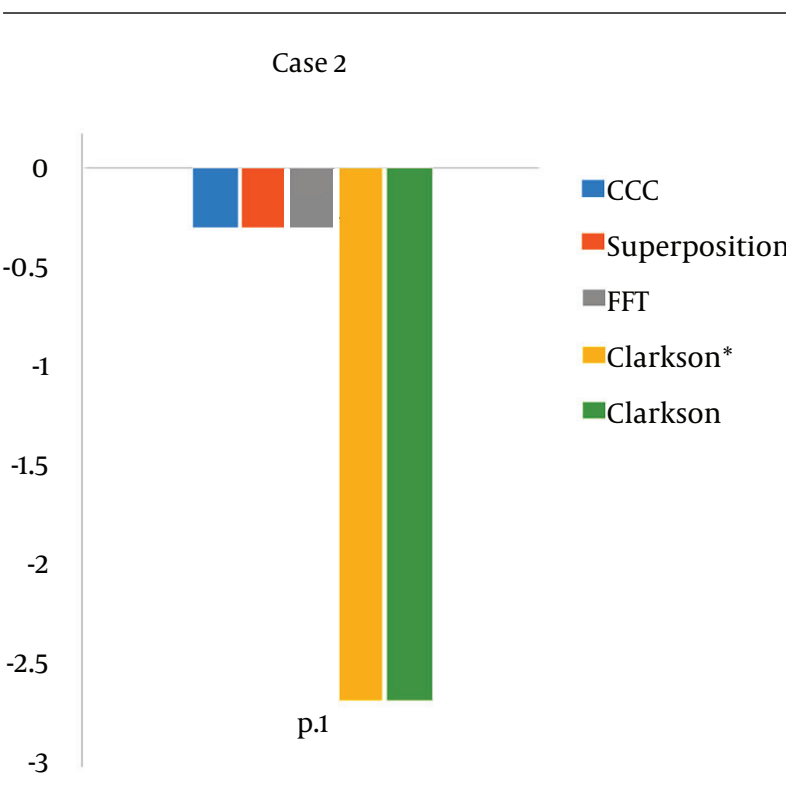

Figure 4. Difference Between Calculation and Measurement in Point 1-Calculated by Different Algorithms in Case Test 2

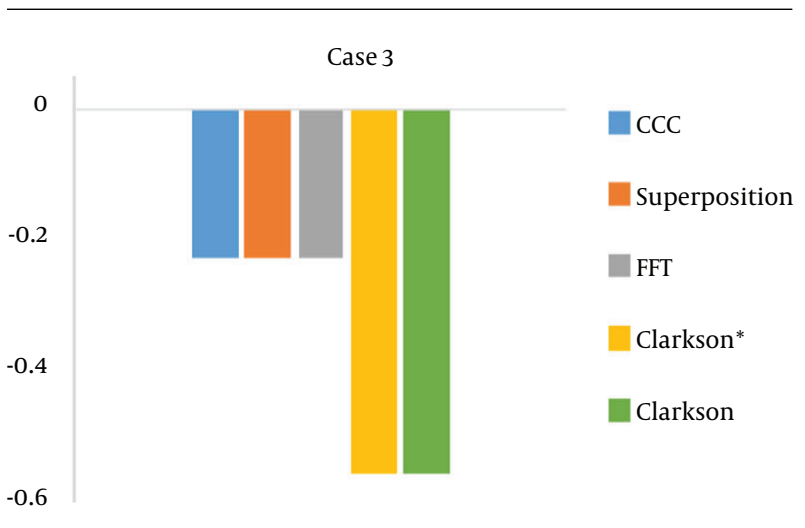

Figure 5. Difference Between Calculation and Measurement in Point 3 Calculated by Different Algorithms in Case Test 3

beams. According to the measurements the Collapsed con, superposition and FFT then Clarkson were accurate, and in some tests, Clarkson failed. The mount of $(\mathrm{MU} / \mathrm{D})=1.23$ for Clarkson and for point kernels that were model-based was 1.25 , the amount MU for the model-base was equal, but the dose calculations in points 6,10 were different. Accuracy for Collapsed con and Superposition and FFT were close and acceptable, but for Clarkson it was different and for some measurements were higher than the criteria agreement. For the L-shape field (case 6), all algorithms passed, in other words, the amount of error was within the criteria agreement, except for point 7 (in the lunge equivalent material) for algorithm FFT, it was above criteria agree- ment and failed. Three points were tested, one of them was in plastic water (point 3), where all the algorithms passed and another point was in a lung equivalent media (point 7), where FFT and Clarkson* failed (agreement criteria was $4 \%$ ). The last point (point 10) was in bone equivalent media, and all of the algorithms passed. In the asymmetrical wedge field (case 7) for model-based algorithms (Superposition, CCC and FFT) the amounts of calculated and measured dose were approximately equal and the amount of error was very small. But for Clarkson, the measured dose was lower than the calculated dose and indicates that Clarkson overestimates the dose, however, Clarkson* underestimates the dose in this case.

In case 8: Deviation outside the agreement criteria was found for Clarkson, and the dose calculation overestimates. For model-based algorithms, in spite of this, the range of agreement was underestimated. For the lateral field, Clarkson and Clarkson* had error that was beyond the agreement criteria.

The result for algorithms being tested for energy of 6 MV is shown in the histogram, and the percentage of results outside of the agreement criteria for all algorithms are shown.

\section{Discussion}

\subsection{Phantom}

This study utilized a heterogeneous phantom designed to represent lung, bone and tissue, utilizing equivalent material based on IAEA methodology and previous studies of this phantom. The phantom can be easily shared and transported between hospitals. It is easy to setup and use for testing. More details of the suitability of different phantoms for these types of tests are given $(17,18)$.

\subsection{TPS Dose Calculation Algorithms}

The accuracy and quality of TPS depends on the type of algorithms utilized in every step of TPS and has been the topic of numerous studies (19). The results for a homogeneous media comply between measurement and calculated dose. The maximum difference results were: Superposition algorithm (-1.7), CCC (1.8), FFT (-2.1) and for Clarkson and Clarkson* was equal to 3.14 , for lateral beam the maximum difference for Superposition was 2.9 and for CCC, FFT, Clarkson and Clarkson* was 3.1, 2.9, 4.7, 6.9 in case test 8, respectively. Except for Clarkson and Clarkson* in the lateral field, the other algorithms passed. Aarup et al. reported good agreement for five algorithms that were tested (20). Clarkson is a simple algorithm that assumes a photon scatter interaction medium. However, it does not include any change in scatter radiation and electron 


\section{Case 4}

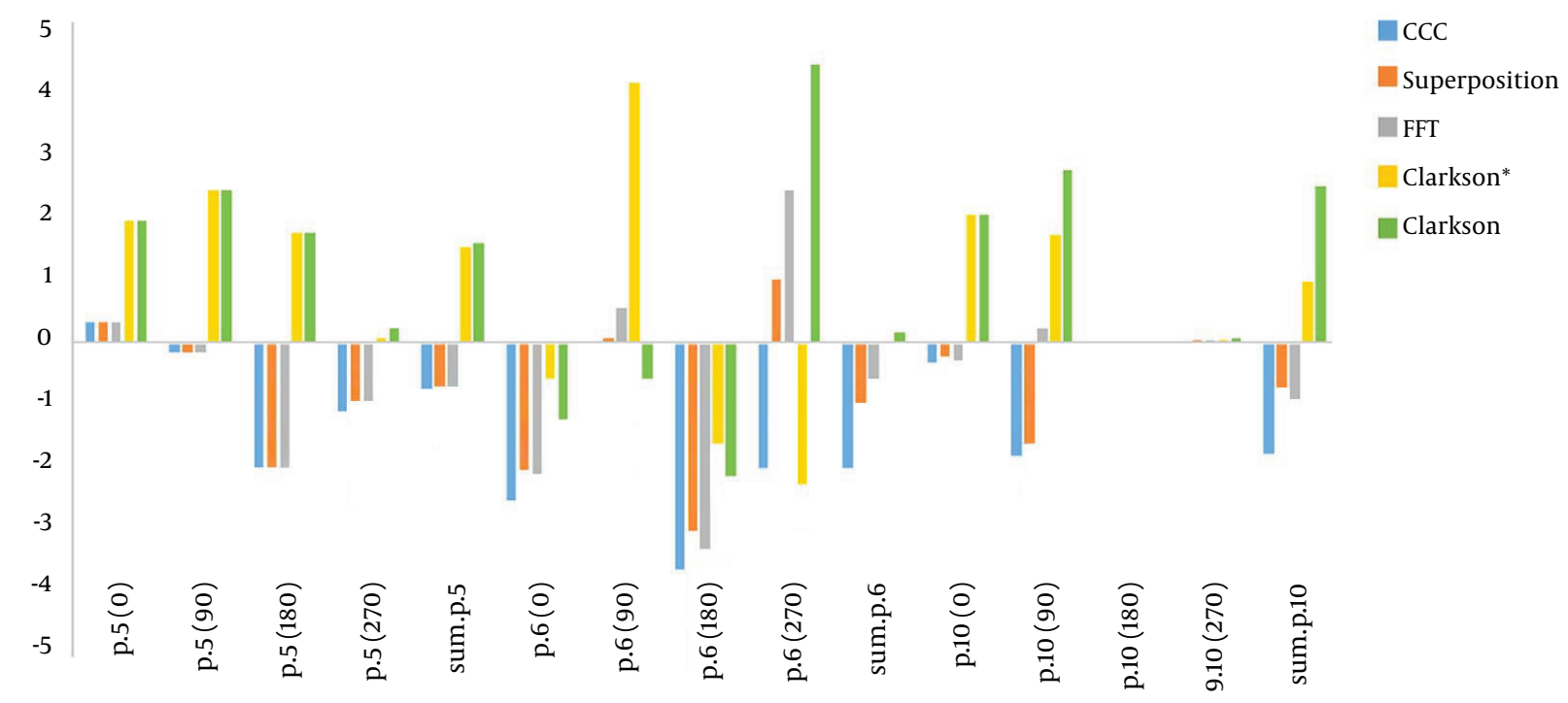

Figure 6. Difference Between Calculation and Measurement Points (5, 6,10 and Sum Dose for Every Point) by Different Algorithms in Case 4

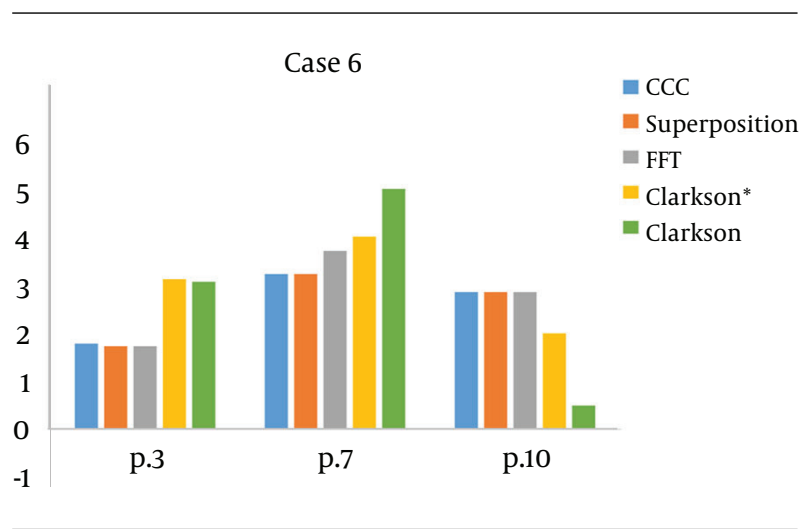

Figure 7. Difference Between Calculations and Measurement by Different Algorithms in Case 6

transport in a heterogeneous medium and it cannot consider a linear process due to insufficient accuracy in this algorithm (21). It is acceptable for clinical applications in the cranial and pelvis lesions (22). For our test, although Clarkson had the most diversions, it can be used for clinical tests. A maximum difference calculation dose was seen (3.17\%) for equivalent bone material and (4.11\%) for equivalent lung material, which underestimates the doses calculated. In the study by Ambolt et al. the Clarkson algorithm has an acceptable degree of agreement between calculations and measurement (23). Linthout et al. also showed good agreement between dose distribution and measured

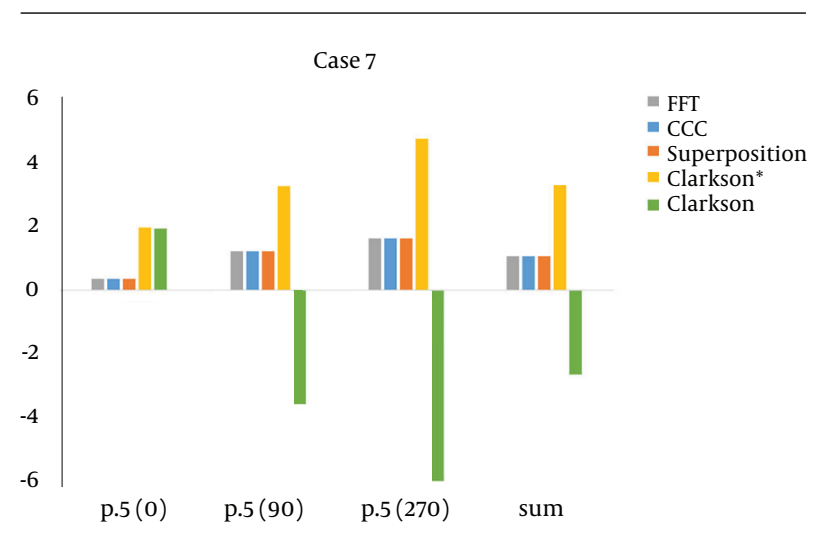

$\overline{\text { Figure 8. Difference Between Calculations and Measurement by Different Algo- }}$ rithms in Case 7

dose by Clarkson algorithm, but for a tissue-air interface the dose calculated by Clarkson overestimates by $12 \%$ (24). In test 2 that uses a tangential beam, it calculated an overestimated dose compared to dose measurement. There was a difference $(-2.5 \%)$ between measurement and calculated dose. The CCC algorithm predicts a dose near to inhomogeneity exactly (25-28). The CCC algorithm as used for the Monte Carlo calculation method and the results show that it has an accuracy of $1 \%$ in complex geometries (29). The CCC algorithm had reasonable speed and accuracy in dose calculation (30). One of the limitations in the accuracy CCC 


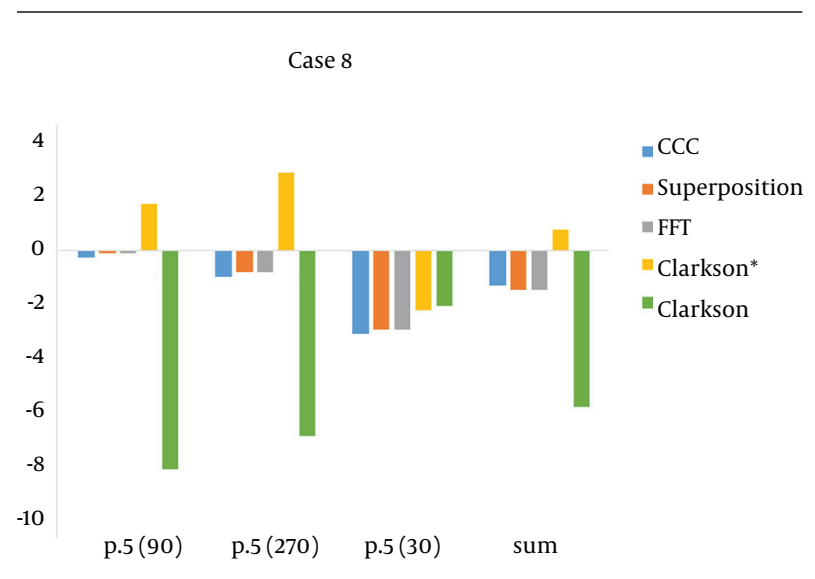

Figure 9. Difference Between Calculations and Measurement by Different Algorithms in Case 8

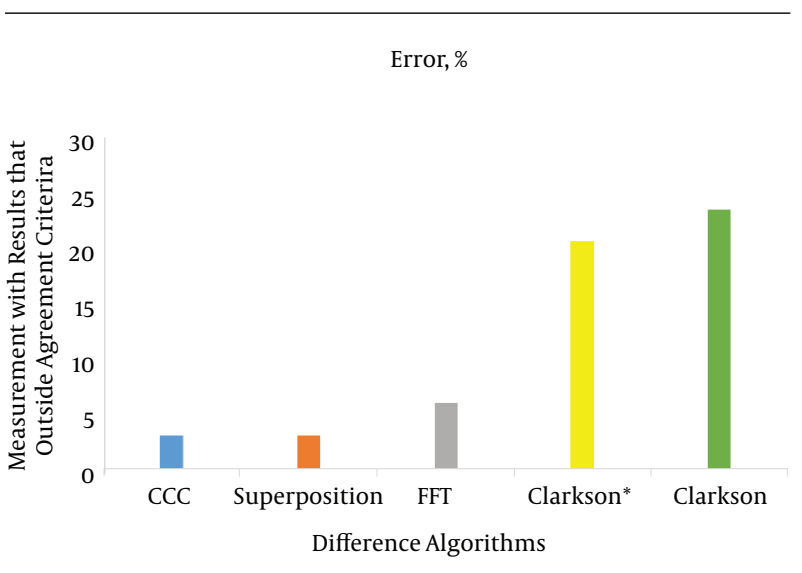

Figure 10. Percentage of Measurements Resulting Out-Side Criteria agreement for Algorithms

is found in a lateral multiple scatter dose (8). The results show that the CCC algorithm predicts in accordance between dose calculation and measurement. Maximum difference was seen in a lung equivalent material (-3.9\%) and for a bone equivalent material it was $-\mathbf{2 . 9 \%}$, where both results overestimate. Asnaashari showed model-based algorithms have better compliance than measurement-based algorithms (31). Linthout et al. showed the three different calculation algorithms (Clarkson, PB and the Collapsed Cone (CC) algorithms) overestimate the dose in the PTV at the boundary with the low-density tissue, with 12, 10 and $7 \%$ for the Clarkson, the PB and the CC algorithm, respectively (24). In another study, Das et al. showed that the Collapsed cone algorithm has better accuracy than the PB algorithm in the interface of inhomogeneous media (27). Nisbet et al. showed Collapsed cone has better accuracy than the PB model in interface lung/tissue and Collapsed cone and the PB model also have inadequate accuracy in interface bone/tissue (25). The maximum deviation was 3.2\% for the Sc algorithm in a lung region. Zhang et al. reported that superposition has better accuracy between FFTC and Superposition in the field edge and heterogeneous media (32). In our study FFTC had 3.8\% maximum deviation for a lung equivalent media. Vicente et al. showed a 9.7\% difference between measured and calculated in cork media with a 6 MV beam by the Superposition algorithm (33). Rutonjski et al. reported a mean 6\% deviation with the Superposition algorithm (34). Scholz et al. showed that the Superposition algorithm had a mean $3 \%$ difference in various fields (35).

This study applied the methodology described in IAEA TECDOC1583 to perform a TPS audit and evaluate dose calculation algorithms. Maximum deviation was found for the Clarkson algorithm with active heterogeneity correction as a field of interest in (ROI) contouring, and error is reduce. Model-based algorithms had better accuracy, and collapsed cone and Superposition were better. These algorithms help to calculate dose with a higher accuracy.

\section{Footnotes}

Authors' Contribution Asiyeh Golestani: study concept and design, acquisition of data, analysis and interpretation of data; Ahmad Mostaar: study concept and design, acquisition of data, study supervision; Mohammad Houshyari: critical revision of the manuscript, Study supervision, technical and material support; Ali Jabbari Arfaie: critical revision of the manuscript for important intellectual content.

Funding/Support This project was supported by Shahid Beheshti University of Medical Sciences as MSc thesis.

\section{References}

1. Shalek RJ. Determination of Absorbed Dose in a Patient Irradiated by Beams of X or Gamma Rays in Radiotherapy Procedures: ICRU Report 24. 24. USA: International Commission on Radiation Units; 1976.

2. Brahme A. Accuracy Requirements and Quality Assurance of External Beam Therapy with Photons and Electrons. United Kingdom: Acta Oncologica; 1988.

3. Brenner DJ. Dose, volume, and tumor-control predictions in radiotherapy. Int J Radiat Oncol Biol Phys. 1993;26(1):171-9. [PubMed: 8482624].

4. Goitein M. Nonstandard deviations. Med Phys. 1983;10(5):709-11. doi: 10.1118/1.595409. [PubMed: 6646083].

5. Mijnheer BJ, Battermann JJ, Wambersie A. What degree of accuracy is required and can be achieved in photon and neutron therapy?. Radiother Oncol. 1987;8(3):237-52. [PubMed: 3107087].

6. Brenner DJ. Reply to: Precision and accuracy in radiotherapy. Radiotherapy and Oncology. 1989;14(2):163-7. doi: 10.1016/01678140(89)90061-3. 
7. Sellakumar P, Arun C, Sanjay SS, Ramesh SB. Comparison of monitor units calculated by radiotherapy treatment planning system and an independent monitor unit verification software. Phys Med. 2011;27(1):21-9. doi: 10.1016/j.ejmp.2010.01.006. [PubMed: 20172756].

8. Ahnesjo A. Collapsed cone convolution of radiant energy for photon dose calculation in heterogeneous media. Med Phys. 1989;16(4):57792. doi: 10.1118/1.596360. [PubMed: 2770632].

9. Francescon P, Cavedon C, Reccanello S, Cora S. Photon dose calculation of a three-dimensional treatment planning system compared to the Monte Carlo code BEAM. Med Phys. 2000;27(7):1579-87. doi: 10.1118/1.599024. [PubMed:10947261].

10. Fippel M. Fast Monte Carlo dose calculation for photon beams based on the VMC electron algorithm. Med Phys. 1999;26(8):1466-75. doi: 10.1118/1.598676. [PubMed: 10501045].

11. Ahnesjo A, Aspradakis MM. Dose calculations for external photon beams in radiotherapy. Phys Med Biol. 1999;44(11):99-155. [PubMed: 10588277].

12. Fippel M, Haryanto F, Dohm O, Nusslin F, Kriesen S. A virtual photon energy fluence model for Monte Carlo dose calculation. Med Phys. 2003;30(3):301-11. doi: 10.1118/1.1543152. [PubMed: 12674229].

13. Vatnitsky S. Commissioning of Radiotherapy Treatment Planning Systems: Testing for Typical External Beam Treatment Techniques. Vienna: International Atomic Energy Agency; 2008.

14. Papanikolaou N, Battista JJ, Boyer AL. Tissue inhomogeneity corrections for megavoltage photon beams. 65. Virginia: AAPM report; 2004.

15. IAEA (International Atomic Energy Agency) . Absorbed dose determination in external beam radiotherapy: an international code of practice for dosimetry based on standards of absorbed dose to water: Technical Report Series 398. Vienna: IAEA; 2000.

16. IAEA (International Atomic Energy Agency) . Commissioning and quality assurance of computerized planning systems for radiation treatment of cancer: Technical Report Series 430. Vienna: International Atomic Energy Agency; 2005.

17. Knoos T, Wieslander E, Cozzi L, Brink C, Fogliata A, Albers D, et al. Comparison of dose calculation algorithms for treatment planning in external photon beam therapy for clinical situations. Phys Med Biol. 2006;51(22):5785-807. doi:10.1088/0031-9155/51/22/005. [PubMed: 17068365].

18. Gershkevitsh E, Schmidt R, Velez G, Miller D, Korf E, Yip F, et al. Dosimetric verification of radiotherapy treatment planning systems: results of IAEA pilot study. Radiother Oncol. 2008;89(3):338-46. doi: 10.1016/j.radonc.2008.07.007. [PubMed:18701178].

19. Van Dyk J. Quality assurance of radiation therapy planning systems: current status and remaining challenges. Int J Radiat Oncol Biol Phys. 2008;71(1 Suppl):S23-7. doi: 10.1016/j.ijrobp.2007.04.095. [PubMed: 18406932].

20. Aarup LR, Nahum AE, Zacharatou C, Juhler-Nottrup T, Knoos T, Nystrom $\mathrm{H}$, et al. The effect of different lung densities on the accuracy of various radiotherapy dose calculation methods: implications for tumour coverage. Radiother Oncol. 2009;91(3):405-14. doi: 10.1016/j.radonc.2009.01.008. [PubMed: 19297051].

21. Khan FM. In: The physics of radiation therapy. 1 ed. Stamathis G, Pine JJW, editors. Baltimore: Williams and Wilkins; 1984. pp. 178-80.Dose calculation in irregular fields-Clarkson's method.

22. Miften MM, Beavis AW, Marks LB. Influence of dose calculation model on treatment plan evaluation in conformal radiotherapy: a threecase study. Med Dosim. 2002;27(1):51-7. [PubMed: 12019966].

23. Ambolt P, Bowden J, Cooper M, Henry A, McCormack S, Morgan A, et al. Commissioning a Superposition Dose Calculation Model for $6 \mathrm{MV}$ Photon Beams on a Radiotherapy Treatment Planning System. J Clin Oncol. 2007;19(3):S40. doi: 10.1016/j.clon.2007.01.399.

24. Linthout N, Verellen D, Van Acker S, Voordeckers M, Bretz A, Storme G. Evaluation of dose calculation algorithms for dynamic arc treatments of head and neck tumors. Radiother Oncol. 2002;64(1):85-95. [PubMed: 12208579].

25. Nisbet A, Beange I, Vollmar HS, Irvine C, Morgan A, Thwaites DI Dosimetric verification of a commercial collapsed cone algorithm in simulated clinical situations. Radiother Oncol. 2004;73(1):79-88. doi 10.1016/j.radonc.2004.06.007. [PubMed:15465150].

26. Weber L, Nilsson P. Verification of dose calculations with a clinical treatment planning system based on a point kernel dose engine. $J$ Appl Clin Med Phys. 2002;3(2):73-87. doi: 10.1120/1.1451983. [PubMed: 11958648].

27. Das IJ, Kase KR, Meigooni AS, Khan FM, Werner BL. Validity of transition-zone dosimetry at high atomic number interfaces in megavoltage photon beams. Med Phys. 1990;17(1):10-6. doi: 10.1118/1.596553. [PubMed: 2106608].

28. Irvine C, Morgan A, Crellin A, Nisbet A, Beange I. The clinical implications of the collapsed cone planning algorithm. Clin Oncol. 2004;16(2):148-54. [PubMed: 15074739].

29. Wang L, Yorke E, Chui CS. Monte Carlo evaluation of tissue inhomogeneity effects in the treatment of the head and neck. Int J Radiat Oncol Biol Phys. 2001;50(5):1339-49. [PubMed: 11483347].

30. Battista JJ, Sharpe MB. True three-dimensional dose computations for megavoltage $x$-ray therapy: a role for the superposition principle.Australas Phys Eng Sci Med. 1992;15(4):159-78. [PubMed: 1482336].

31. Asnaashari K, Nodehi MR, Mahdavi SR, Gholami S, Khosravi HR. Dosimetric comparison of different inhomogeneity correction algorithms for external photon beam dose calculations. J Med Phys. 2013;38(2):74-81. doi: 10.4103/0971-6203.111310. [PubMed: 23776310].

32. Zhang $\mathrm{P}$, Simon A, De Crevoisier R, Haigron P, Nassef $M H$, Li $\mathrm{B}$, et al. A new pencil beam model for photon dose calculations in heterogeneous media. Phys Med. 2014;30(7):765-73. doi: 10.1016/j.ejmp.2014.05.010. [PubMed: 24954922].

33. Garcia-Vicente F, Minambres A, Jerez I, Modolell I, Perez L, Torres J. Experimental validation tests of fast Fourier transform convolution and multigrid superposition algorithms for dose calculation in lowdensity media. Radiother Oncol. 2003;67(2):239-49. doi: 10.1016/s0167 8140(03)00037-9.

34. Rutonjski L, Petrovic B, Baucal M, Teodorovic M, Cudic O, Gershkevitsh $\mathrm{E}$, et al. Dosimetric verification of radiotherapy treatment planning systems in Serbia: national audit. Radiat Oncol. 2012;7:155-63. doi: 10.1186/1748-717X-7-155. [PubMed: 22971539].

35. Scholz C, Schulze C, Oelfke U, Bortfeld T. Development and clinical application of a fast superposition algorithm in radiation therapy. $R a$ diother Oncol. 2003;69(1):79-90. [PubMed: 14597360]. 